\title{
Psychophysiological correlates of face processing in social phobia
}

\author{
Iris-Tatjana Kolassa ${ }^{a, *}$, Wolfgang H.R. Miltner ${ }^{b}$ \\ ${ }^{a}$ Clinical Psychology and Neuropsychology, University of Konstanz, Germany \\ ${ }^{b}$ Biological and Clinical Psychology, Friedrich Schiller University Jena, Germany
}

\section{A R T I C L E I N F O}

\section{Article history:}

Accepted 6 August 2006

Available online 12 September 2006

Keywords:

Anxiety disorder

Emotional Stroop

Event related potentials (ERPs)

Heart rate

Face processing

Social phobia

\begin{abstract}
A B S T R A C T
Social phobia has been associated with abnormal processing of angry faces, which directly signal disapproval a situation that social phobics fear. This study investigated the electrophysiological correlates of emotional face processing in socially phobic and non phobic individuals. Subjects identified either the gender (modified emotional Stroop task) or the expression of angry, happy, or neutral faces. Social phobics showed no deviations from controls in reaction times, heart rates, P1, or P2 amplitudes in response to angry faces, although elevated FSS scores were associated with higher P1 amplitudes in social phobic persons. In addition, social phobic persons showed enhanced right temporo parietal N170 amplitudes in response to angry faces in the emotion identification task. Furthermore, higher scores on the Social Phobia and Anxiety Inventory (SPAI) were associated as a trend with larger N170 amplitudes in response to angry faces in the emotion identification task. Thus, the present results suggest that social phobics show abnormalities in the early visual processing of angry faces, as reflected by the enhanced right hemispheric N170 when the emotion of the angry face was the focus of attention, while behavioral responses and heart rates showed no evidence for preferred processing of angry facial expressions.
\end{abstract}

\section{Introduction}

Social phobia is a highly prevalent disorder characterized by extreme fear of negative evaluation in social interaction and performance situations. People with social phobia fear that they will do or say something embarrassing, be negatively evaluated, or have their excessive anxiety symptoms noticed. Anxiety symptoms manifest themselves in physiological symptoms such as shaking, blushing or sweating, or in behavioral symptoms such as stuttering, poor eye contact, mumbling, nail biting, or a trembling voice (Albano, 1995; Beidel and Turner, 1998).

Cognitive models of anxiety (e.g., Eysenck, 1997; Rapee and Heimberg, 1997; Williams et al., 1997) have suggested that anxiety patients selectively attend to threat cues, i.e., they exhibit an attentional bias. One specific threat cue in the social sphere is an angry face, which signals to the individual that his/her behavior violated social rules or social expectations (see Averill, 1982), a situation that people with social phobia fear. Thus, the question whether social phobic patients show

\footnotetext{
* Corresponding author. Department of Psychology, Clinical and Neuropsychology, University of Konstanz, PO Box 5560, D25, 78457 Konstanz, Germany. Fax: +49 7531884601.

E mail address: Iris.Kolassa@uni konstanz.de (I. T. Kolassa).
} 
anomalies in face processing has become a research topic in recent years.

One of the most commonly used paradigms to study attentional biases in phobia is the emotional Stroop para digm (MacLeod, 1991; Wells and Matthews, 1994; Williams et al., 1996), a modified version of the original Stroop task (Stroop, 1935). In the emotional Stroop paradigm, the color of words or pictures varying in personal emotional significance has to be identified either verbally or manually, e.g., by pushing a button. The phenomenon that threatening stimu lus attributes impair the processing of non threatening stimulus characteristics has been called emotional interference. The difference in color naming latencies between anxiety related and neutral stimuli the emotional Stroop effect provides a measure of the attentional bias towards emotional stimuli. Several studies using emotional Stroop tasks found evidence for an attentional bias to social threat words in patients with social phobia (Becker et al., 2001; Hope et al., 1990; Lundh and Öst, 1996; Maidenberg et al., 1996; Mattia et al., 1993; Spector et al., 2003).

To our knowledge, all emotional Stroop paradigms to date have used social threat words for investigating the attentional bias in social phobia. However, since emotional facial expres sions directly signal approval or disapproval, it has been proposed that facial expressions of emotion are more ecolo gically valid stimuli than words (see discussion in Gilboa Schechtman et al., 1999) and should thus lead to a greater attentional bias in social phobic persons.

Pictures of different emotional facial expressions have been used in several research paradigms other than the emotional Stroop to investigate attentional biases in social phobia (e.g., Chen et al., 2002; Gilboa Schechtman et al., 1999; Mansell et al., 1999; Mogg et al., 2004). For example, in a visual search paradigm, a so called "face in the crowd para digm", persons with generalized social phobia and non anxious controls both detected angry faces faster than happy ones in a neutral crowd, but individuals with social phobia showed an even larger enhancement than controls (Gilboa Schechtman et al., 1999). Functional magnetic resonance imaging (fMRI) studies have shown an increased difference in BOLD response in the amygdala to angry and contemptuous than to happy (Stein et al., 2002) as well as neutral faces (Birbaumer et al., 1998) in social phobic patients than in controls. In addition, Straube et al. (2004) also found a stronger activation of the insula in social phobics than in controls in response to angry compared to neutral schematic and photographic faces, independent of whether subjects had to identify the expression of the face or the type of stimulus (photographic or schematic). Furthermore, when subjects were asked to identify the type of stimulus, phobic individuals exhibited stronger activations in response to angry than to neutral photographic faces in the insula, the right amygdala, the parahippocampal gyrus, the fusiform gyrus, and the superior temporal sulcus compared to controls. Thus, phobic individuals more intensively pro cessed angry facial expressions than controls even when this was not required according to task instructions.

Abnormalities in the emotional and structural processing of facial expressions can be reflected in early visual event related potentials such as the occipital P1 and the posterior occipito temporal N170 component. The P1 is known to be an attention sensitive component (for a review see Mangun, 1995) and is also modulated by facial emotion (Batty and Taylor, 2003) as well as a person's affective judgment of face images as liked or disliked (Pizzagalli et al., 1999). This judgment dependent modulation may be caused by re entrant processes from more anterior structures such as the amygdala, which are involved in attentional and motivational processes (Amaral, 2003). If persons with social phobia show an early bias towards angry facial expressions, this should therefore be reflected in larger P1 amplitudes in response to angry faces compared to controls.

The bilateral posterior occipito temporal N170 component is thought to originate from neuronal networks in ventral occipito temporal structures in the vicinity of the occipito temporal sulcus (Schweinberger et al., 2002; compare also Henson et al., 2003). Several studies have suggested it to be a face specific ERP component, which is elicited by faces but not by other stimuli such as animal faces, human hands, cars, or items of furniture (Bentin et al., 1996; for a qualification, see Schweinberger et al., 2004). Additionally, it has been demon strated that this component is linked to late stages of structural encoding, where representations of global face configurations are generated in order to be utilized by subsequent face recognition processes (Eimer, 2000). The N170 is sensitive to emotional expressions (Batty and Taylor, 2003; Caharel et al., 2005; Pizzagalli et al., 2000; Williams et al., 2006; however, see Holmes et al., 2003; Schupp et al., 2004). For example, Caharel et al. (2005) reported larger N170 amplitudes for emotional expressions of disgust than for neutral or smiling faces. Batty and Taylor (2003) reported larger N170 amplitudes to fearful than to neutral, happy, disgusted, surprised, sad, and angry faces. Using linked mastoids instead of an average reference, Williams et al. (2006) observed larger N170 amplitudes for emotional (fearful and happy) compared to neutral faces. Whereas several studies showed an emo tional modulation of the N170 amplitude, it is still unclear whether individuals with social phobia show abnormalities in the early processing of negative facial expressions, reflected for example in enhanced N170 amplitudes to angry faces.

Based on the above observations that social phobics show anomalies in the encoding of angry faces, this study aimed at investigating the attentional bias for angry faces in indivi duals with social phobia by measuring reaction times, heart rates, and event related potentials in response to emotional faces. Social phobic patients, spider phobic individuals as a clinical control group, and healthy controls saw angry, happy, and neutral looking faces. Subjects' task was either to identify the gender of the face (modified emotional Stroop paradigm) or to identify the facial expression (emotion classification task) by pushing a button. The expectation was to find greater emotional Stroop interference (expressed as longer response latencies) for angry faces during the gender identification task in socially anxious than in socially non anxious subjects. For the emotion classification task, faster reactions to angry faces than to happy or neutral ones were expected in all groups, with even faster reactions by social phobic than by spider phobic or control subjects. Finally, it was hypothesized that larger early visual event related potentials (P1 and N170) and larger decelerations in 
Table 1 - Mean reaction times (M) and standard deviations (SD) for the Identification of gender and emotion of pictures of angry, happy, and neutral faces

\begin{tabular}{|c|c|c|c|c|c|c|}
\hline \multirow[t]{2}{*}{ Condition } & \multicolumn{2}{|c|}{ Controls } & \multicolumn{2}{|c|}{$\begin{array}{c}\text { Social } \\
\text { phobics }\end{array}$} & \multicolumn{2}{|c|}{$\begin{array}{c}\text { Spider } \\
\text { phobics }\end{array}$} \\
\hline & M & SD & $\mathrm{M}$ & SD & $\mathrm{M}$ & $\mathrm{SD}$ \\
\hline \multicolumn{7}{|c|}{ Gender identification } \\
\hline Angry & 590.85 & 74.35 & 606.59 & 72.04 & 572.89 & 62.11 \\
\hline Happy & 589.06 & 71.06 & 604.16 & 76.13 & 579.98 & 59.13 \\
\hline Neutral & 575.75 & 78.61 & 592.23 & 74.72 & 554.27 & 49.59 \\
\hline \multicolumn{7}{|c|}{ Emotion identification } \\
\hline Angry & 731.46 & 66.79 & 735.49 & 61.30 & 701.79 & 61.36 \\
\hline Happy & 696.36 & 82.79 & 700.54 & 68.43 & 684.08 & 70.58 \\
\hline Neutral & 724.28 & 70.85 & 732.23 & 58.88 & 694.03 & 62.94 \\
\hline
\end{tabular}

heart rates would be found in social phobic subjects when processing angry facial expressions, reflecting their atten tional bias towards threatening stimuli.

\section{Results}

\subsection{Behavioral data and reaction times}

Overall, subjects failed to respond on $5.3 \%$ of the trials, and incorrect responses were observed on $3.7 \%$ of the trials. There was no difference between groups in total errors (omissions and incorrect choices), Kruskal Wallis $\chi^{2}(2, n$ 57) 0.13 , p 0.94 .

Response latencies were longer for emotion than for gender identification, $F(1,54) 386.70, p<0.0001$ (cf. Table 1). Furthermore, an interaction of Task $\times$ Emotion, $F(2,216) \quad 9.51$, $p$ 0.0001, was observed. In the gender identification task, subjects identified the gender of neutral faces faster than the gender of emotional faces, $p \quad 0.007$. In the emotion identifica tion task, subjects identified happy facial expressions faster than neutral or angry facial expressions, happy vs. angry, $p$ 0.0006, happy vs. neutral, $p$ 0.0004, in contrast to our hypothesis. No interaction of Group $\times$ Task $\times$ Emotion was observed, again in contrast to our hypothesis, as well as no other interaction with Group.

\subsection{Heart rates}

Groups did not differ in baseline heart rates ( $5000 \mathrm{~ms}$ ). The heart rate showed the typical orienting response, main effect of Time, $F(5,275) 45.51, p<0.0001$, and was modulated by facial emotion, $F(2,106)$ 4.21, p 0.02 (cf. Fig. 1). Contrasts revealed that angry faces led to significantly larger decelera tions than neutral faces, $p$ 0.005. The comparison happy neutral failed to be significant, $p$ 0.10, and the contrast happy angry was not significant. The emotion identification task led to a more pronounced orienting response than the gender identification task, interaction of TimexTask, $F(5,1507) \quad 2.52, p \quad 0.03$. No significant interaction with Group was observed.

\subsection{Electrophysiological data}

Three components were analyzed (Fig. 2): (a) posterior occipital P1, (b) posterior temporal N170, and (c) posterior occipital P2.

\subsubsection{P1 amplitude and latency}

Although spider phobic individuals showed slightly larger P1 amplitudes than controls and social phobic persons, there was no significant main effect of Group, $F(2,50) 2.45, p 0.10$. Emotional faces led to larger P1 amplitudes than neutral faces (see Fig. 3), $F(2,100)$ 4.81, $p$ 0.01. Simple contrasts revealed that both angry and happy faces led to larger P1 amplitudes than neutral faces, $p \quad 0.003$ (angry neutral) and p 0.03 (happy neutral). Although angry faces led to slightly larger amplitudes than happy faces, this difference was not significant. In addition, P1 amplitudes were larger over the right than the left hemisphere, $F(1,52) \quad 7.64, p \quad 0.008$. Analysis of P1 latency revealed neither significant main effects nor interactions.

ANCOVAs were calculated separately for each group with questionnaire values as a covariate. For persons with social phobia, a significant relationship between FSS values and P1

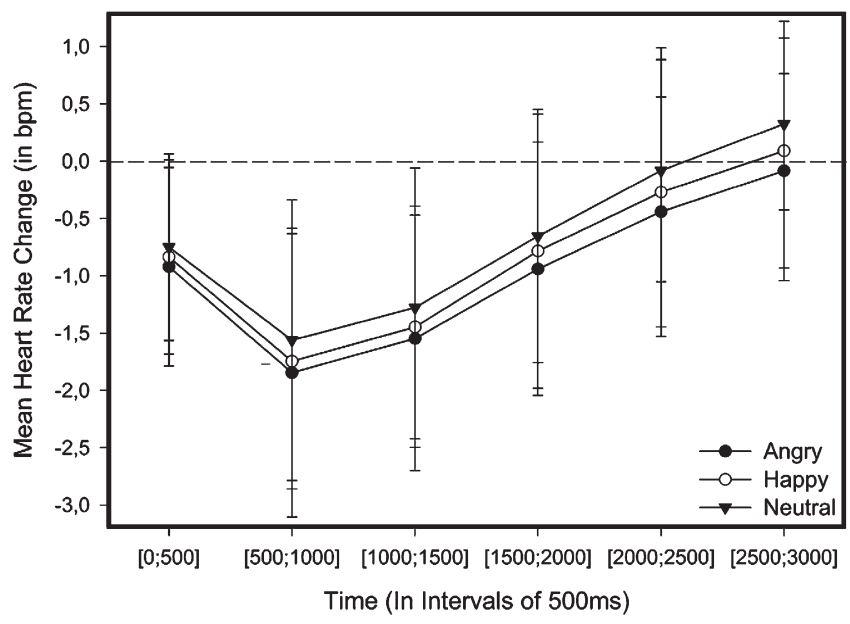

Fig. 1 - Mean heart rate changes (and SD) in beats per minute (bpm) in time intervals of 500 ms in response to angry, happy, and neutral faces. 

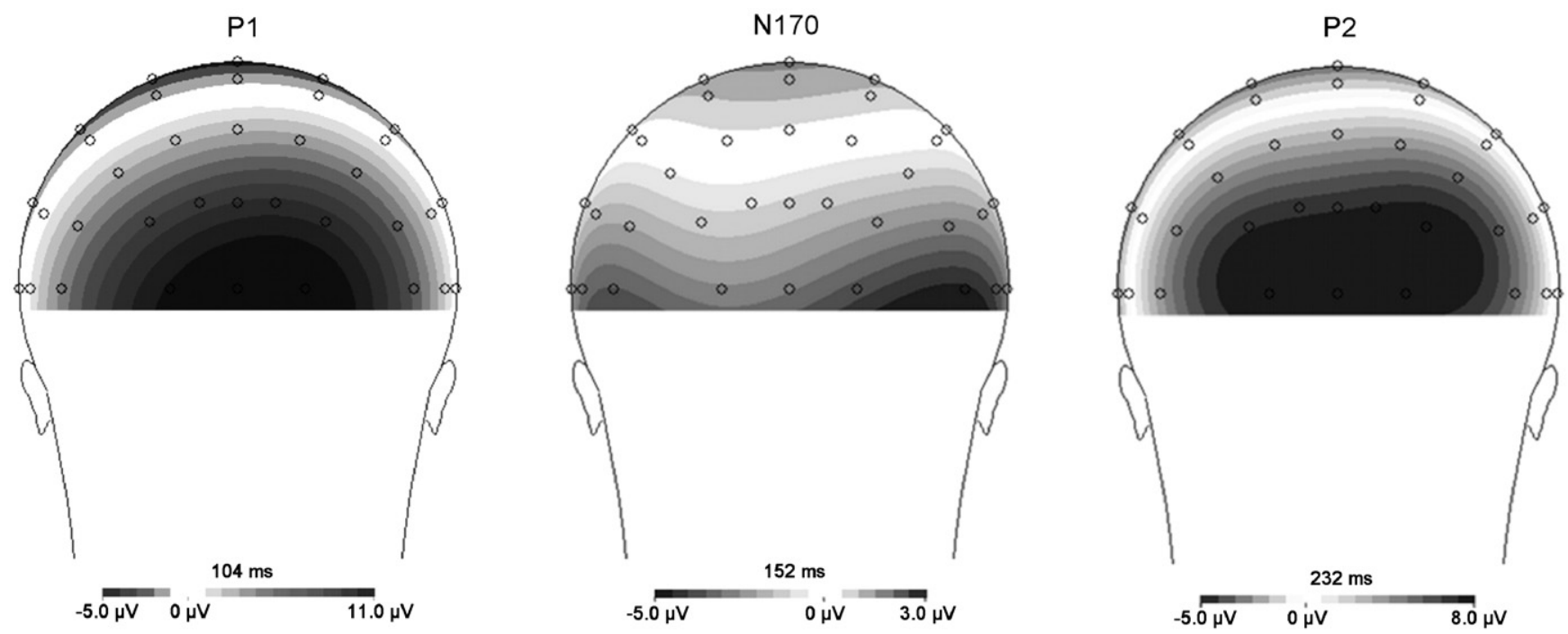

Fig. 2 - Scalp potential maps reveal the topography of early visual ERP components: the P1 component (left) shows an occipital distribution, the N170 component (middle) a bilateral posterior-temporal distribution, and the P2 component (right) a more anterior-occipital topography.

amplitude was observed, $F(1,122) \quad 6.69, p \quad 0.01$; the larger the score on the FSS, the larger the P1 amplitude. No such relationship was present in the spider phobia or control group; however, both these groups had significantly lower FSS scores than the social phobia group.

\subsubsection{N170 amplitude and latency}

N170 amplitudes were modulated by the emotionality of the facial expression, $F(2,100) \quad 5.27, p 0.007$ (see Fig. 4). Simple contrasts revealed significantly higher N170 amplitudes for happy compared to angry faces, $p \quad 0.004$, and compared to
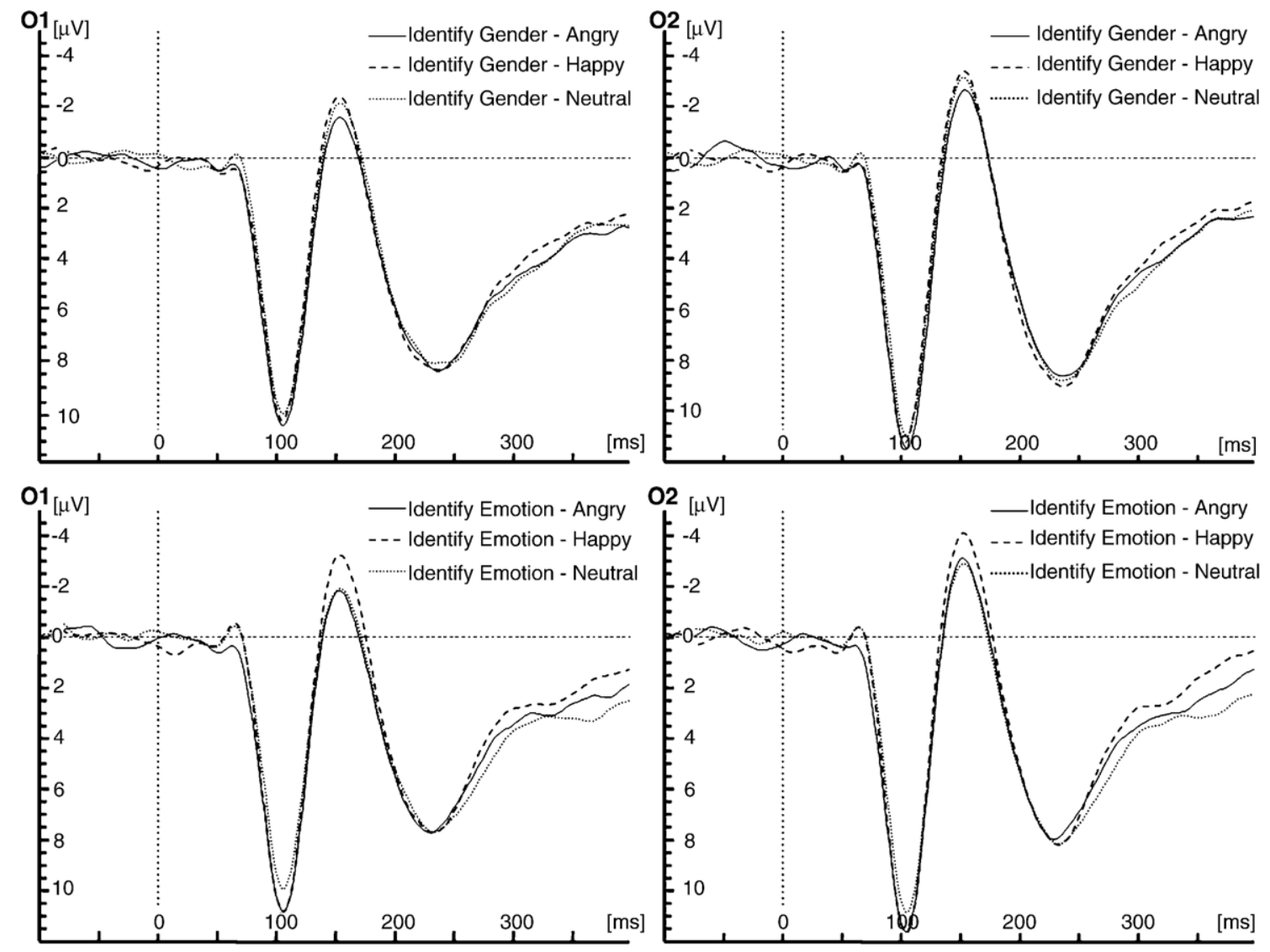

Fig. 3 - Grand mean ERPs for the gender (upper row) and emotion identification (lower row) of angry, happy, and neutral faces at electrodes 01 (left) and 02 (right). 

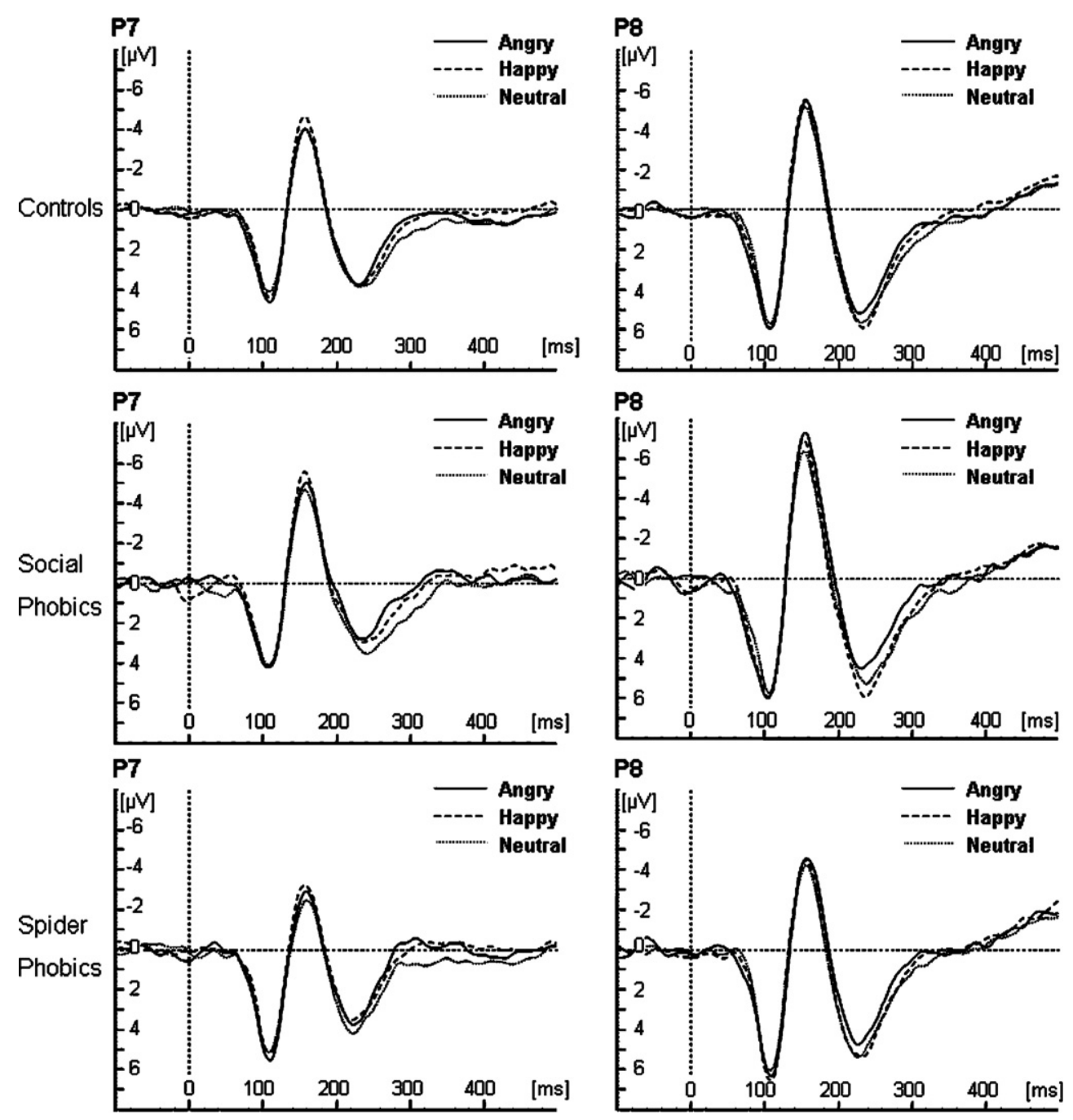

Fig. 4 - Grand mean ERPs in response to angry, happy, and neutral faces for controls (upper row), social phobics (mid row), and spider phobics (lower row) at electrodes P7 (left) and P8 (right).

neutral faces, $p \quad 0.008$, while there was no significant differ ence between neutral and angry faces. In addition, the effect of emotion on the N170 amplitude was less pronounced in the gender identification task than in the emotion identification task, interaction of Task $\times$ Emotion, $F(2,100) 3.30, p 0.04$. Emotional (angry and happy) faces led to larger N170 amplitudes in the emotion identification than in the gender identification task, $p \quad 0.01$.

Furthermore, N170 amplitudes were larger over right compared to left sites, $F(1,50) \quad 6.62, p \quad 0.01$, modulated by an interaction of Laterality $\times$ Emotion, $F(2,100)$ 3.63, $p \quad 0.03$. Happy faces led to larger N170 amplitudes compared to neutral and angry faces over the left hemisphere, $p \quad 0.0003$, but not over the right hemisphere.

Finally, the influence of emotion differed between groups, Group $\times$ Emotion interaction, $F(4,100)$ 2.80, $p$ 0.03. However, the contrast which tested the hypothesis that social phobics show larger N170 amplitudes in response to angry faces compared to spider phobic persons and controls failed significance, $p \quad 0.09$. Yet, when identifying the emotion of an angry face, social phobic individuals showed larger N170 amplitudes in response to angry faces compared to controls and spider phobics over the right hemisphere, $p \quad 0.05$ (cf. Fig. 5). Correlations between N170 amplitudes in the emotion identification task and SPAI scores revealed a trend for a negative relationship, $r \quad 0.35, p \quad 0.08$; the larger the SPAI score the larger (i.e., more negative) the N170 amplitude in response to angry faces over right hemispheric sites. Such a relationship was not present in the spider phobic and control group.

Analysis of N170 latency revealed no significant main effect of Group or interaction with Group. There was a trend for happy faces to be associated with shorter N170 latencies in the gender identification task than angry and neutral faces, interaction of Task $\times$ Emotion, $F(2,100) \quad 2.74, p \quad 0.07$.

\subsubsection{P2 amplitude and latency}

A main effect of Task, $F(1,50) \quad 9.31, p \quad 0.004$, and of Laterality, $F(1,52) \quad 5.96, p$ 0.02, was observed. Larger P2 amplitudes were observed over the right compared to the left hemi sphere, and gender identification led to larger P2 amplitudes than emotion identification. Furthermore, trends for a main 


\section{Emotion Identification of Angry Faces}
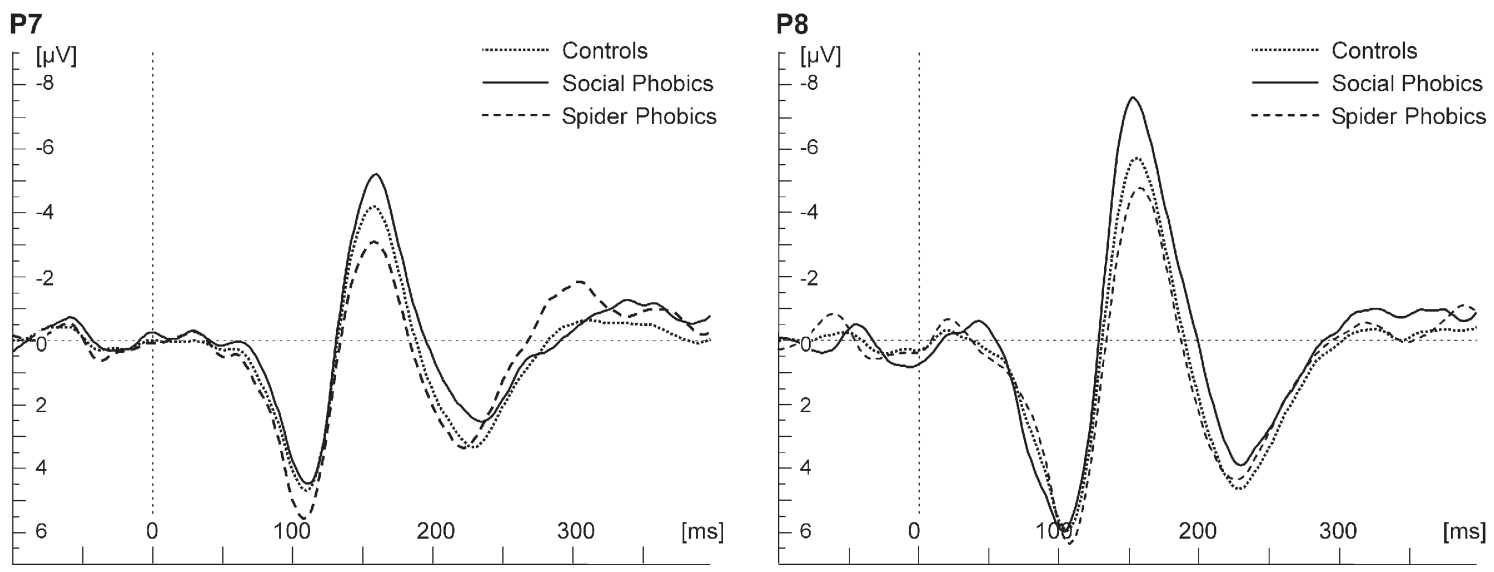

Fig. 5 - Grand mean ERPs when identifying the emotion of angry faces at electrodes P7 (left) and P8 (right) for controls, social phobic, and spider phobic individuals.

effect of Emotion, $F(2,100)$ 2.71, $p \quad 0.07$, and an interaction of Emotion $\times$ Task, $F(2,100) \quad 2.77, p \quad 0.07$, were observed.

Analysis of P2 latency revealed a main effect of Task, $F(1,50) \quad 13.58, p \quad 0.0006$. Longer P2 amplitudes were observed for gender compared to emotion identification. In addition, a trend for longer P2 latencies in social phobics compared to the control groups was observed, $F(2,50) \quad 2.72, p \quad 0.08$.

\section{Discussion}

This study found evidence that individuals with social phobia differ from healthy control subjects in the processing of angry faces: social phobic persons showed larger N170 amplitudes over right temporo parietal sites when identifying the emo tion of an angry face. Furthermore, higher scores on social anxiety as measured by the SPAI were associated as a tendency with larger N170 amplitudes in response to angry faces over right temporo parietal sites.

In contrast, the early visual P1 amplitude did not differ between social phobic, spider phobic, and non phobic indivi duals in response to angry faces, although it was larger in response to emotional compared to neutral faces. In addition, higher FSS scores were associated with higher P1 amplitudes in social phobics.

Heart rates and reaction times revealed no anomalies in the processing of angry faces in individuals with social phobia. Social phobic subjects showed neither a defense response nor even an enhanced orienting response compared to controls in response to angry faces. Instead, the orienting response in all groups varied with facial emotion, i.e., it was larger for angry compared to neutral faces and non significantly larger for happy compared to neutral faces.

The expected emotional interference in patients with social phobia when identifying the gender of an angry face was not observed. Instead, all subjects identified the gender of neutral faces significantly faster than the gender of emotional faces, suggesting that the emotionality of a facial expression captures attention and slows down performance of the primary task, i.e., the gender identification task. Furthermore, in the emotion focused task, the expected general response time advantage for angry faces was not observed. Instead, all groups identified happy faces significantly faster than angry or neutral ones.

\subsection{Emotional modulation of the face-specific N170 component}

In accordance with recent studies by Batty and Taylor (2003) and Caharel et al. (2005), this study found a modulation of the N170 by facial emotion. This finding is, however, in contrast to some previous studies (Eimer and Holmes, 2002; Eimer et al., 2003; Holmes et al., 2003; Schupp et al., 2004), which found no modulation of the N170 component by facial emotion. Several reasons might account for these conflicting findings: first, in the earlier studies either the facial emotion was not task relevant (Eimer and Holmes, 2002; Holmes et al., 2003; Schupp et al., 2004), or second, although it was task relevant, a different experimental design was used: the study design of Eimer et al. (2003) only allowed peripheral attention to facial emotion, possibly reducing emotional modulation of the N170. Accordingly, the present study found less emotional modula tion of N170 in the gender than the emotion identification task. These results present an interesting contrast to earlier reports suggesting that the N170 is relatively unaffected by task demands (gender vs. expression judgments, Schyns et al., 2003), and is hardly affected by attention (Cauquil et al., 2000). This issue deserves further investigation.

Furthermore, social phobic participants showed larger right hemispheric N170 amplitudes in response to angry faces when identifying the emotion of an angry face than the control groups. This is to our knowledge the first study showing abnormalities in the processing of angry faces in social phobia in ERP components related to face processing. However, the finding is in accordance with previous fMRI studies showing abnormal processing of angry faces in social phobia (e.g., Birbaumer et al., 1998; Stein et al., 2002; Straube et al., 2004). The results suggest that angry faces may be processed more intensely by social phobics than controls, leading to increased activation in a number of brain regions. 
For example, Straube et al. (2004) found increased activation of the amygdala, the parahippocampal gyrus, and the extra striate visual cortex in an implicit identification task and increased activation of the insula both during an implicit and an explicit identification task in response to angry faces compared to neutral faces. Yet, the difficulties involved in assigning ERPs as measured in EEG to generating structures as investigated by fMRI preclude a final integration of EEG and fMRI data on differential processing of phobic stimuli. In addition, further studies are necessary to elucidate which stage of face processing is altered in social phobia. Does the enhanced N170 reflect differences in attention, intensity of emotional or structural processing, or simply expertise in the processing of angry faces?

Finally, consistent with a valence specific hemispheric asymmetry for processing emotional stimuli (see Canli, 1999 for a review), emotional modulation of the N170 amplitude was dependent on the hemisphere: happy faces led to larger N170 amplitudes compared to neutral and angry faces over the left hemisphere, but not over the right hemisphere. In addition, larger right hemispheric N170 amplitudes were observed in response to angry faces in social phobics. These results are in accordance with a valence specific hemispheric asymmetry observed by Jansari et al. (2000), who used an emotion discrimination task in which a neutral stimulus was always paired with an emotional morphed stimulus (happi ness, surprise, fear, anger, disgust, or sadness). Jansari et al. (2000) found that subjects performed better in discriminating positive expressions when the emotional face was to the right, whereas when discriminating negative emotional faces, subjects performed better when the emotional face was to the left of the neutral face.

\subsection{Influence of emotionality on P1 amplitude}

The emotion dependent modulation of the P1 confirms that emotional perception and judgment can take place very early in the visual stream (Öhman and Soares, 1994), presumably due to re entrant processes from the amygdala (Amaral, 2003). The present results are in agreement with previous findings showing an emotional modulation of P1 amplitude (Batty and Taylor, 2003) as well as a modulation of P1 by personal affective judgment of faces (Pizzagalli et al., 1999). Because the P1 is thought to be attention sensitive (for a review see Mangun, 1995), the present data suggest that emotional faces received more attentional processing than neutral faces. However, Keil et al. (2002), using pictures taken from the IAPS (Lang et al., 1997), found no P1 enhancement by affective content, which might be due to different stimuli (non face pictures) or to different task instructions (instead of performing a classification task, subjects only had to look at the stimuli). In addition, low level visual stimulus differences between emotional and neutral faces might account, at least to some extent, for the emotional modulation of the P1. However, a study by Delplanque et al. (2004) also found a modulation of early P1 by the emotional valence of stimuli taken from the IAPS. In this study, subjects had the explicit task to press a button when they saw an emotionally pleasant, unpleasant, or neutral picture in a series of standard checker board stimuli.

\subsection{Effect of emotionality on heart rates}

Phobic subjects typically show a defense reflex in response to their feared object, characterized by a temporary cardiac deceleration followed by an acceleration (Fredrikson, 1981; Globisch et al., 1999; Hamm et al., 1997; Hare and Blevings, 1975). However, the present study found neither a more pronounced orienting response nor a defense response to angry faces in social phobic persons. Correspondingly, by measuring skin conductance responses, Merckelbach et al. (1989) found no evidence that social phobics are particularly sensitive to negative facial cues. Thus, angry faces are not feared stimuli for social phobic persons in the same way as spiders are feared stimuli for spider phobic individuals (Kolassa et al., 2005; Miltner et al., 2005). Yet, heart rates were modulated by facial emotion, replicating findings by Codispoti et al. (2001): in all groups, neutral pictures elicited the weakest and unpleasant pictures the strongest orienting response, with pleasant pictures in between.

\subsection{Absence of emotional Stroop interference in social phobic subjects}

Emotional Stroop interference reported in social phobics when color naming social threat words (Becker et al., 2001; Hope et al., 1990; Lundh and Öst, 1996; Maidenberg et al., 1996; Mattia et al., 1993; Spector et al., 2003) was not replicated with angry faces. Yet, present results are in line with a recent study by Kolassa et al. (2005), who also found no emotional Stroop interference in spider phobic subjects when color naming spider pictures. Presumably, angry faces either are not specifically related to the concerns of social phobics, or facial expressions do not raise semantic associations to emotional concerns of social phobic patients, in contrast to emotional words related to likely concerns or relevant threats (Mathews and Klug, 1993), and thus do not cause interference.

Compared to neutral faces, angry and happy faces slowed down performance in the gender identification task. P1 amplitude varied corresponding with facial emotionality, i.e., P1 was larger for emotional (angry and happy) compared to neutral faces. Thus, it is plausible to assume that emotional faces activated limbic structures such as the amygdala, and via re entrant projections from the amygdala back to the occipital cortex (e.g., Lang et al., 1998) led to increased attentional processing of these stimuli, slowing down perfor mance in the gender identification task.

\subsection{An advantage for happy faces in the emotion identification task}

The faster detection of happy faces when subjects' task was to identify the emotion of a facial expression seems in conflict with previous studies showing advantageous processing of negative faces or fear relevant stimuli as compared to positive faces or neutral objects, respectively (e.g., Fox et al., 2000; Hansen and Hansen, 1988; Öhman et al., 2001; Tipples et al., 2002). Yet, several studies reported faster categorization of happy faces than expressions of anger (Billings et al., 1993; Harrison et al., 1990; Hugdahl et al., 1993; Kaufmann and Schweinberger, 2004; Leppänen et al., 2003), disgust (Leppänen 
et al., 2003; Stalans and Wedding, 1985), sadness (Kirita and Endo, 1995; Stanners et al., 1985), or emotionally neutral faces (Hugdahl et al., 1993; Schweinberger et al., 2003). The con flicting findings may be due to different task designs: whereas visual search paradigms show faster detection of threatening stimuli, emotion recognition tasks involving a choice reaction time task report an advantage for happy faces.

Several explanations for this happy face advantage have been proposed: First, happy faces contain less overlapping character istics with other facial emotions (see Johnston et al., 2001), and happiness may be conveyed by the single facial feature of a smiling mouth (see Adolphs, 2002). Second, happy faces are encountered more often than other facial expressions in every day life (Bond and Siddle, 1996), possibly leading to more efficient processing (as argued in Öhman et al., 2001) due to higher baseline activation of relevant processing units (see McClelland and Rumelhart, 1981) or stronger associative links for high frequency stimuli (as suggested by McClelland, 1979). Evidence for an advantage for positive words (e.g., Feyereisen et al., 1986) and positive non face pictures (Lehr et al., 1966) also supports a general advantage for processing positive stimuli.

In conclusion, the present results suggest that social phobics show abnormalities in the processing of angry faces, as reflected in the enhanced right hemispheric N170 when the emotion of the angry face was the focus of attention. On the other hand, social phobics did not deviate from controls in P1 amplitude, heart rates, or response time pattern to angry faces. In particular, no emotional interference for angry faces was observed in social phobics, contrary to previous findings with social threat words. Angry faces, in contrast to social threat words, may not raise semantic associations to emotional concerns of social phobic patients and thus may cause no interference. Social phobic subjects' response pattern thus suggests that angry faces are not feared stimuli for them in the same way spiders are feared stimuli for spider phobics.

\section{Experimental procedures}

\subsection{Pilot study}

In a pilot study, 12 individuals with social phobia $(5$ male, 7 female), 14 with spider phobia ( 5 male, 9 female), and 15 controls ( 9 male, 6 female) rated the pictures of angry, happy, and neutral faces used in this study, which were taken from the Karolinska Directed Emotional Faces set (KDEF, Lundqvist, Flykt and Öhman, Department of Neurosciences, Karolinska Hospital, Stockholm, Sweden, 1998). Subjects' mean age was 23.6 years, SD 4.1, age range 1733 years. Each picture was rated on affective valence and arousal with an adapted version of the Self Assessment Manikin Scale (SAM; Bradley and Lang, 1994; Lang, 1980). The SAM is a relatively culture free picto graphic assessment instrument which has been used in various emotion studies.

ANOVA revealed a main effect of Emotion for valence, $F$ $(2,76)$ 73.4, $p$ 0.0005, and arousal ratings, $F(2,76)$ 19.49, $p$ 0.0005. Subsequent LSD pairwise comparisons revealed that all groups rated angry faces as significantly more unpleasant and arousing than neutral, both $p \quad 0.0005$, or happy faces, both $p$ 0.0005. In addition, happy faces were
Table 2-Mean valence and arousal ratings $(M)$ and standard deviations (SD) for pictures of angry, happy, and neutral facial expressions for each group

\begin{tabular}{|c|c|c|c|c|c|c|}
\hline \multirow[t]{2}{*}{ Rating } & \multicolumn{2}{|c|}{$\begin{array}{l}\text { Controls } \\
\text { (n 15) }\end{array}$} & \multicolumn{2}{|c|}{$\begin{array}{l}\text { Social } \\
\text { phobics } \\
\left(\begin{array}{ll}n & 12\end{array}\right)\end{array}$} & \multicolumn{2}{|c|}{$\begin{array}{l}\text { Spider } \\
\text { phobics } \\
\left(\begin{array}{ll}n & 14\end{array}\right)\end{array}$} \\
\hline & $\mathrm{M}$ & SD & M & SD & M & SD \\
\hline \multicolumn{7}{|l|}{ Valence } \\
\hline Angry & 3.31 & 1.20 & 2.74 & 0.84 & 3.21 & 1.62 \\
\hline Happy & 5.11 & 1.38 & 5.09 & 0.99 & 5.33 & 1.12 \\
\hline Neutral & 4.18 & 0.80 & 3.98 & 0.99 & 4.26 & 1.20 \\
\hline \multicolumn{7}{|l|}{ Arousal } \\
\hline Angry & 2.61 & 1.77 & 3.44 & 1.45 & 2.81 & 1.49 \\
\hline Happy & 2.19 & 1.62 & 2.41 & 1.50 & 2.01 & 1.14 \\
\hline Neutral & 1.65 & 1.33 & 2.35 & 1.09 & 2.14 & 1.26 \\
\hline
\end{tabular}

Note. The original scale (Lang, 1980; Bradley and Lang, 1994) was slightly modified: the SAM scale ranged from 0 to 8 with $0=$ highly unpleasant/low arousing and $8=$ highly pleasant/highly arousing.

rated significantly more pleasant than neutral faces, $p \quad 0.0005$, but equally (low) arousing. Patients with social phobia did not deviate from this general pattern (see Table 2).

\subsection{Subjects}

Fifty seven subjects (mean age 23.2 years, SD 3.4 , age range 1932 years) participated in the study: 19 social phobic subjects (10 male, 9 female), 19 spider phobic subjects (9 male, 10 female), and 19 non phobic controls (10 male, 9 female). A subset of the participants had also participated in the pilot study $(7$ social phobics, 5 spider phobics, and 9 controls). Groups did not differ significantly regarding age. Fifty four participants were right handed and 3 left handed, as measured by the Edinburgh handedness questionnaire (Oldfield, 1971). Participants were the same as in Kolassa et al. (2005). All participants were students of the University of Jena.

Subjects were recruited through local newspaper adver tisement and within the university student population. All participants provided informed consent, and the procedures were approved by the ethics committee of the University of Jena. Subjects were paid 6 Euro per hour for their participation. As an additional compensation, social phobic patients were offered a 10 session group social skills training after the experiment (Hinsch and Pfingsten, 2002), and spider phobic individuals could participate in a one day spider phobia treatment (Öst, 1989).

Subjects were free of neurological disorders, a history of seizures, current psychotropic medication, or a history of previous mental disorders. Participants were screened with the Structured Clinical Interview for DSM IV (SCID I; Wittchen et al., 1997). Social phobic and spider phobic individuals were included in the study if they fulfilled DSM IV (American Psychiatric Association, 1994) criteria for social phobia or spider phobia, respectively, but had no history or current symptoms of other major Axis I disorders. Controls were accepted if they had no current or previous history of major disorders according to DSM IV. 
Subjects completed the Social Phobia and Anxiety Inven tory (SPAI; Fydrich, 2002; Turner et al., 1989), the Spider Questionnaire (SPQ; Klorman et al., 1974), the Beck Depression Inventory (BDI; Hautzinger et al., 1995), the State Trait Anxiety Inventory (STAI; Laux et al., 1981), and the Fear Survey Schedule (FSS; Wolpe and Lang, 1964). See Table 3 for mean questionnaire values and standard deviations.

\subsection{Stimuli and paradigm}

One hundred and twenty pictures were taken from the KDEF picture set. The experiment was divided into two blocks, each preceded by a training phase. In each block, subjects saw 60 emotional faces in a randomized order (20 of each emotional category with an equal number of male and female faces). Subjects' task was to identify either the gender or the facial expression of the presented face by pressing a button with the index finger of the dominant hand. The order of the two con ditions as well as the sequence of keys which had to be pressed to classify the gender or expression was randomized across subjects. Stimuli were shown for $1 \mathrm{~s}$ with a variable inter stimulus interval of $23 \mathrm{~s}$.

\subsection{Assessment and analysis of EEG}

During the recording session, subjects sat in a comfortable chair in a sound attenuated room. Stimuli were presented on a 20 inch Sony monitor (resolution $800 \times 600$ ) placed $1.1 \mathrm{~m}$ in front of the subject's eyes. EEG was measured with a 62 channel montage (easy cap, Falk Minow Systems, Germany) according to the international 1010 system (Chatrian et al., 1998) with $\mathrm{Cz}$ as a reference electrode. Electrode impedances were kept below $5 \mathrm{k} \Omega$. Vertical and horizontal electrooculo grams (vEOG and hEOG) were measured for later correction of eye movements and blink artifacts. All signals were continu ously sampled in AC mode at a rate of $500 \mathrm{~Hz}$ (gain 1000, filters: high pass $0.05 \mathrm{~Hz}$, low pass $70 \mathrm{~Hz}$ ).

The EEG data were filtered offline (low pass $30 \mathrm{~Hz}$, high pass $0.1 \mathrm{~Hz}, 24 \mathrm{~dB}$ /oct both), segmented ( $200 \mathrm{~ms}$ to $900 \mathrm{~ms}$ ), corrected for blinks (Gratton et al., 1983), and screened for artifacts using the software Brain Vision Analyzer 1.04 (Brain Products $\mathrm{GmbH}$, Germany). Trials with artifacts (amplitude deviations of $\pm 150 \mu \mathrm{V}$ ) were excluded from analysis. The averages for each condition and for each subject were aligned to a $200 \mathrm{~ms}$ pre stimulus baseline and rereferenced to an average reference in order to reduce the bias resulting from the choice of the reference sensor. The data of 1 social phobic subject (male) were excluded from further analysis because of extreme alpha activity. The data of 3 control subjects ( 2 male, 1 female) were excluded because it was impossible to detect P1 and/or N170 components.

Through artifact removal, $3.2 \%$ of trials were excluded. Separate average waveforms were calculated for angry, happy, and neutral faces for the two different tasks. Three compo nents were identified and further analyzed (Fig. 2): (a) poster ior occipital P1 peaking around $100 \mathrm{~ms}$, (b) posterior occipital P2 peaking around $230 \mathrm{~ms}$, and (c) posterior temporal N170 peaking around $160 \mathrm{~ms}$. Peaks were detected semiautomati cally: P1 (70 $140 \mathrm{~ms}$ ) at electrodes O1 and O2, N170 (120 $190 \mathrm{~ms}$ ) and P2 (210 $280 \mathrm{~ms}$ ) both at electrodes P7 and P8.

\subsection{Assessment and analysis of heart rate data}

Heart rate and respiration were recorded using DASYLab 5.0 (Datalog GmbH, Germany). The electrocardiogram (ECG) was measured by 2 precordial leads (Ag/AgCl electrodes at V3 and V4) according to Wilson et al. (1944). A reference electrode (V3R) was placed at the corresponding right side of V3. A fourth ground electrode was attached to the right waist. Electrode sites were cleaned with alcohol to improve impedances. Amplification of ECG was $0.5 \mathrm{mV} / \mathrm{V}$. The data were high pass filtered at $0.53 \mathrm{~Hz}$ and low pass filtered at $30 \mathrm{~Hz}$. Respiration was measured by an elastic belt containing a piezoelectrical element, placed at the abdomen (male) or the chest (female). Amplification was $5 \mathrm{mV} / \mathrm{V}$, high pass filter $10 \mathrm{~Hz}$, and low pass filter $1 \mathrm{~Hz}$.

The heart rate data were analyzed with Brain Vision Analyzer 1.04. By visual inspection of the data, it was assured that respiration was not stimulus locked. Therefore, the influence of respiration on heart rate (respiratory sinus arrhythmia) is reduced by averaging. Heart rates were determined by $\mathrm{R}$ wave detection and subsequent conversion into beats per minute (bpm) in $500 \mathrm{~ms}$ intervals between $500 \mathrm{~ms}$ pre and $3000 \mathrm{~ms}$ post stimulus. Heart rate changes were computed by subtracting the $500 \mathrm{~ms}$ pre stimulus baseline from the post stimulus heart rates. Averaging these

Table 3 - Mean questionnaire values (M) and standard deviations (SD) for each group

\begin{tabular}{|c|c|c|c|c|c|c|c|}
\hline \multirow[t]{2}{*}{ Questionnaire } & \multicolumn{2}{|c|}{$\begin{array}{l}\text { Control group } \\
\left(\begin{array}{ll}n & 19\end{array}\right)\end{array}$} & \multicolumn{2}{|c|}{$\begin{array}{l}\text { Social phobics } \\
\text { (n 19) }\end{array}$} & \multicolumn{2}{|c|}{$\begin{array}{l}\text { Spider phobics } \\
\qquad\left(\begin{array}{l}n \\
19\end{array}\right)\end{array}$} & \multirow[t]{2}{*}{ One way ANOVA } \\
\hline & M & SD & M & $\mathrm{SD}$ & M & SD & \\
\hline SPAI & 33.53 & 16.89 & 126.80 & 18.17 & 43.90 & 15.25 & $F(2,54)=175.68, p<0.0001^{\mathrm{a}, \mathrm{b}}$ \\
\hline STAI T & 30.79 & 5.92 & 50.47 & 6.61 & 33.32 & 7.86 & $F(2,54)=46.52, p<0.0001^{a, b}$ \\
\hline $\mathrm{BDI}$ & 2.68 & 2.71 & 9.42 & 7.09 & 4.79 & 4.67 & $F(2,54)=8.52, p<0.001^{a, b}$ \\
\hline SPQ & 2.47 & 1.78 & 2.58 & 1.95 & 20.89 & 2.87 & $F(2,54)=422.44, p<0.0001^{b, c}$ \\
\hline FSS & 22.16 & 12.33 & 63.42 & 30.95 & 40.68 & 20.47 & $F(2,54)=12.52, p<0.0001^{\mathrm{a}, \mathrm{b}}$ \\
\hline
\end{tabular}

Note. The German scores of the Social Phobia and Anxiety Inventory (SPAI) were transformed into the original scores (Turner et al., 1989). STAI T, State Trait Anxiety Inventory Trait Version; BDI, Beck Depression Inventory; SPQ Spider Questionnaire; FSS, Fear Survey Schedule.

${ }^{a}$ Control group is different from social phobics $(p<0.05)$.

b Spider phobics are different from social phobics $(p<0.05)$.

c Control group is different from spider phobics $(p<0.05)$. 
heart rates per time interval over stimuli is a well established procedure (Graham, 1978).

\subsection{Statistical analysis}

For data analysis, linear mixed effects models (Laird and Ware, 1982) were implemented using SAS 9.1 (SAS Institute Inc.). In all analyses of variance (ANOVAs) and analyses of covariance (ANCOVAs), subjects served as a random effect, whereas all other factors were fixed effects. Significant effects in an ANOVA were further analyzed by calculating appropriate contrasts in SAS, where rejection of the null hypothesis was controlled by Holm's sequential rejection algorithm (Holm, 1979). Original $p$ values that remained significant after $\alpha$ correction are reported above.

For the analysis of reaction times, trials were excluded in which no reaction time button press occurred, the answer was wrong, the reaction time was below $200 \mathrm{~ms}$ or longer than 2 SD from the individual mean. To examine the influence of task and facial expression on reaction times, a $3 \times 2 \times 3$ ANOVA with between factor Group and repeated measures factors Task (identify gender, identify expression) and Emotion (angry, happy, neutral) was performed. For the analysis of heart rates, a $3 \times 2 \times 3 \times 6$ repeated measures ANOVA with between factor Group and repeated measures factors Task, Emotion, and Time (in intervals of $500 \mathrm{~ms}$ ) was calculated. To analyze P1, N170, and P2 amplitudes, a $3 \times 2 \times 2 \times 3$ ANOVA with between factor Group and repeated measures factors Laterality (left, right), Task, and Emotion was performed.

\section{Acknowledgments}

This study was funded by the German Research Foundation (Deutsche Forschungsgemeinschaft) grant Mi265/6 1 awarded to Wolfgang H.R. Miltner. We would also like to thank the German National Academic Foundation (Studienstiftung des deutschen Volkes) for awarding a doctoral grant to Iris Tatjana Kolassa. Thanks are also due to Stephan Kolassa, Sandra Riske, Alexander Mohr, and Katharina Stößel for their assistance in conducting the study.

\section{R E F E R E N C E S}

Adolphs, R., 2002. Recognizing emotion from facial expressions: Psychological and neurological mechanisms. Behav. Cogn. Neurosci. Rev. 1, 2162.

Albano, A.M., 1995. Treatment of social anxiety in adolescents. Cogn. Behav. Pract. 2, 271298.

Amaral, D.G., 2003. The amygdala, social behavior, and anger detection. Ann. N. Y. Acad. Sci. 1000, 337347.

American Psychiatric Association, 1994, Diagnostic and statistical manual of mental disorders (4th ed.). Author, Washington, DC.

Averill, J.R., 1982. Anger and Aggression: An Essay on Emotion. Springer Verlag, New York.

Batty, M., Taylor, M.J., 2003. Early processing of the six basic facial emotional expressions. Cogn. Brain Res. 17, 613620.

Becker, E.S., Rinck, M., Margraf, J., Roth, W.T., 2001. The emotional Stroop effect in anxiety disorders. General emotionality or disorder specificity. J. Anxiety Disord. 15, 147159.
Beidel, D.C., Turner, S.M., 1998. Shy Children, Phobic Adults: Nature and Treatment of Social Phobia. American Psychological Association, Washington, DC.

Bentin, S., Allison, T., Puce, A., Perez, E., McCarthy, G., 1996. Electrophysiological studies of face perception in humans. J. Cogn. Neurosci. 8, 551565.

Billings, L.S., Harrison, D.W., Alden, J.D., 1993. Age differences among women in the functional asymmetry for bias in facial affect perception. Bull. Psychon. Soc. 31, 317320.

Birbaumer, N., Grodd, W., Diedrich, O., Klose, U., Erb, M., Lotze, M., Schneider, F., Weiss, U., Flor, H., 1998. fMRI reveals amygdala activation to human faces in social phobics. NeuroReport 9, 12231226.

Bond, N.W., Siddle, D.A.T., 1996. The preparedness account of social phobia: some data and alternative explanations. In: Rapee, R.M. (Ed.), Current Controversies in the Anxiety Disorders. Guilford Press, London, pp. 291316.

Bradley, M.M., Lang, P.J., 1994. Measuring emotion: The self assessment manikin and the semantic differential. J. Behav. Ther. Exp. Psychiatry 25, 4959.

Caharel, S., Courtay, N., Bernard, C., Lalonde, R., Rebaï, M., 2005. Familiarity and emotional expression influence an early stage of face processing: an electrophysiological study. Brain Cogn. 59, 96100.

Canli, T., 1999. Hemispheric asymmetry in the experience of emotion. Neuroscientist 5, 201207.

Cauquil, A.S., Edmonds, G.E., Taylor, M.J., 2000. Is the face sensitive N170 the only ERP not affected by selective attention? NeuroReport 11, 21672171.

Chatrian, G.E., Lettici, E., Nelson, P.L., 1998. Modified nomenclature for the $10 \%$ electrode system. J. Clin. Neurophysiol. 5, 183186.

Chen, Y.P., Ehlers, A., Clark, D.M., Mansell, W., 2002. Patients with generalized social phobia direct their attention away from faces. Behav. Res. Ther. 40, 677687.

Codispoti, M., Bradley, M.M., Lang, P.J., 2001. Affective reactions to briefly presented pictures. Psychophysiology 38, 474478.

Delplanque, S., Lavoie, M.E., Hot, P., Silvert, L., Sequeira, H., 2004. Modulation of cognitive processing by emotional valence studied through event related potentials in humans. Neurosci. Lett. 356, 14.

Eimer, M., 2000. The face specific N170 component reflects late stages in the structural encoding of faces. NeuroReport 11, 23192324.

Eimer, M., Holmes, A., 2002. An ERP study on the time course of emotional face processing. NeuroReport 13, 15.

Eimer, M., Holmes, A., McGlone, F.P., 2003. The role of spatial attention in the processing of facial expression: An ERP study of rapid brain responses to six basic emotions. Cogn. Affect. Behav. Neurosci. 3, 97110.

Eysenck, M.W., 1997. Anxiety and Cognition: A Unified Theory. Psychology Press, East Sussex, UK.

Feyereisen, P., Malet, C., Martin, Y., 1986. Is the faster processing of expressions of happiness modality specific? In: Ellis, H.D., Jeeves, M.A., Newcombe, F., Young, A. (Eds.), Aspects of Face Processing. Martinus Nijhoff, Boston, pp. 349355.

Fox, E., Lester, V., Russo, R., Bowles, R.J., Pichler, A., Dutton, K., 2000. Facial expressions of emotions: Are angry faces detected more efficiently? Cogn. Emot. 14, 6192.

Fredrikson, M., 1981. Orienting and defensive reactions to phobic and conditioned fear stimuli in phobics and normals. Psychophysiology 18, 456465.

Fydrich, T., 2002. Soziale Phobie und Angst Inventar. In: Brähler, E., Schumacher, J., Strauß, B. (Eds.), Diagnostische Verfahren in der Psychotherapie [Diagnostic methods in psychotherapy]. Hogrefe, Göttingen, pp. 335338.

Gilboa Schechtman, E., Foa, E.B., Amir, N., 1999. Attentional biases for facial expressions in social phobia. The face in the crowd paradigm. Cogn. Emot. 13, 305318. 
Globisch, J., Hamm, A.O., Esteves, F., Öhman, A., 1999. Fear appears fast: temporal course of startle reflex potentiation in animal fearful subjects. Psychophysiology 36, 6675.

Graham, F.K., 1978. Constraints on measuring heart rate and period sequentially through real and cardiac time. Psychophysiology 15, 492495.

Gratton, G., Coles, M.G.H., Donchin, E., 1983. A new method for off line removal of ocular artifact. Electroencephalogr. Clin. Neurophysiol. 55, 468484.

Hamm, A.O., Cuthbert, B.N., Globisch, J., Vaitl, D., 1997. Fear and the startle reflex: Blink modulation and autonomic patterns in animals and mutilation fearful subjects. Psychophysiology 43, 97107.

Hansen, C.H., Hansen, R.D., 1988. Finding the face in the crowd: An anger superiority effect. J. Pers. Soc. Psychol. 54, 917924.

Hare, R.D., Blevings, G., 1975. Defensive responses to phobic stimuli. Biol. Psychol. 3, 113.

Harrison, D.W., Gorelczenko, P.M., Cook, J., 1990. Sex differences in the functional asymmetry for facial affect perception. Int. J. Neurosci. 52, 1116.

Hautzinger, M., Bailer, M., Worall, H., Keller, F., 1995. Beck Depressions Inventar (BDI) [Beck depression inventory], Testhandbuch, 2nd ed. Hans Huber, Bern.

Henson, R.N., Goshen Gottstein, Y., Ganel, T., Otten, L.J., Quayle, A., Rugg, M.D., 2003. Electrophysiological and haemodynamic correlates of face perception, recognition and priming. Cereb. Cortex 13, 793805.

Hinsch, R., Pfingsten, U., 2002. Gruppentraining sozialer Kompetenzen [Group training of social skills]. Beltz, Weinheim.

Holm, S., 1979. A simple sequentially rejective multiple test procedure. Scand. J. Stat. 6, 6570.

Holmes, A., Vuilleumier, P., Eimer, M., 2003. The processing of emotional facial expression is gated by spatial attention: Evidence from event related brain potentials. Brain Res. Cogn. Brain Res. 16, 174184.

Hope, D.A., Rapee, R.M., Heimberg, R.G., Dombeck, M.J., 1990. Representations of the self in social phobia: vulnerability to social threat. Cogn. Ther. Res. 21, 681696.

Hugdahl, K., Iversen, P.M., Jonsen, B.H., 1993. Laterality for facial expressions: does the sex of the subjects interact with the sex of the stimulus face? Cortex 29, 325331.

Jansari, A., Tranel, D., Adolphs, R., 2000. A valence specific lateral bias for discriminating emotional facial expressions in free field. Cogn. Emot. 14, 341353.

Johnston, P.J., Katsikitis, M., Carr, V.J., 2001. A generalized deficit can account for problems in facial emotion recognition in schizophrenia. Biol. Psychol. 58, 203227.

Kaufmann, J.M., Schweinberger, S.R., 2004. Expression influences the recognition of familiar faces. Perception 33, 399408.

Keil, A., Bradley, M.M., Hauk, O., Rockstroh, B., Elbert, T., Lang, P.J., 2002. Large scale neural correlates of affective picture processing. Psychophysiology 39, 641649.

Kirita, T., Endo, M., 1995. Happy face advantage in recognizing facial expressions. Acta Psychol. (Amst.) 89, 149163.

Klorman, R., Weerts, T.C., Hastings, J.E., Melamed, B.G., Lang, P.J., 1974. Psychometric description of some specific fear questionnaires. Behav. Ther. 5, 401409.

Kolassa, I. T., Musial, F., Mohr, A., Trippe, R.H., Miltner, W.H.R., 2005. Electrophysiological correlates of threat processing in spider phobics. Psychophysiology 42, 520530.

Laird, N.M., Ware, J.H., 1982. Random effects models for long itudinal data. Biometrics 38, 963974.

Lang, P.J., 1980. Behavioral treatment and bio behavioral assessment: computer applications. In: Sidowski, J.B., Johnson, J.H., Williams, T.A. (Eds.), Technology in Mental Health Care Delivery Systems. Ablex Publishing, Norwood, NJ, pp. 119135.

Lang, P.J., Bradley, M.M., Cuthbert, B.N., 1997. International Affective Picture System (IAPS): Instruction manual and affective ratings. The Center for Research in Psychophysiology, University of Florida, Gainsville, FL.

Lang, P.J., Bradley, M.M., Fitzsimmons, J.R., Cuthbert, B.N., Scott, J.D., Moulder, B., Nangia, V., 1998. Emotional arousal and activation of the visual cortex: an fMRI analysis. Psychophysiology 35, 199210.

Laux, L., Glanzmann, P., Schaffner, P., Spielberger, C.D., 1981. Das State Trait Angstinventar (STAI G) [The state trait anxiety inventory]. Beltz, Weinheim.

Lehr, D.J., Bergum, B.O., Standing, T.E., 1966. Response latency as a function of stimulus affect and presentation order. Percept. Mot. Skills 23, 11111116.

Leppänen, J.M., Tenhunen, M., Hietanen, J.K., 2003. Faster choice reaction times to positive than to negative facial expressions. J. Psychophysiol. 17, 113123.

Lundh, L. G., Öst, L. G., 1996. Stroop interference, self focus and perfectionism in social phobia. Pers. Individ. Differ. 20, 725731.

MacLeod, C.M., 1991. Half a century research on the Stroop effect: An integrative review. Psychol. Bull. 109, 163203.

Maidenberg, E., Chen, E., Craske, M., Bohn, P., Bystritsky, A., 1996. Specificity of attentional bias in panic disorder and social phobia. J. Anxiety Disord. 10, 529541.

Mangun, G.R., 1995. Neural mechanisms of visual selective attention. Psychophysiology 32, 418.

Mansell, W., Clark, D.M., Ehlers, A., Chen, Y.P., 1999. Social anxiety and attention away from emotional faces. Cogn. Emot. 13, 673690.

Mathews, A., Klug, F., 1993. Emotionality and interference with color naming in anxiety. Behav. Res. Ther. 31, 305313.

Mattia, J.I., Heimberg, R.G., Hope, D.A., 1993. The revised Stroop color naming task in social phobics. Behav. Res. Ther. 31, 305313.

McClelland, J.L., 1979. On the time relations of mental processes: An examination of systems of processes in cascade. Psychol. Rev. 86, 287330.

McClelland, J.L., Rumelhart, D.E., 1981. An interactive activation model of context effects in letter perception: I. An account of basic findings. Psychol. Rev. 88, 375407.

Merckelbach, H., van Hout, van den Hout, M.A., Mersch, P.P., 1989. Psychophysiological and subjective reactions of social phobics and normals to facial stimuli. Behav. Res. Ther. 27, 289294.

Miltner, W.H.R., Trippe, R.H., Krieschel, S., Gutberlet, I., Hecht, H., Weiss, T., 2005. Event related brain potentials and affective responses to threat in spider/snake phobic and non phobic subjects. Int. J. Psychophysiol. 57, 4352.

Mogg, K., Philippot, P., Bradley, B.P., 2004. Selective attention to angry faces in clinical social phobia. J. Abnorm. Psychol. 113, 160165.

Öhman, A., Soares, J.J.F., 1994. Unconscious anxiety: Phobic responses to masked stimuli. J. Abnorm. Psychol. 103, 231240.

Öhman, A., Lundqvist, D., Esteves, F., 2001. The face in the crowd revisited: a threat advantage with schematic stimuli. J. Pers. Soc. Psychol. 80, 381396.

Oldfield, R.C., 1971. The assessment and analysis of handedness: the Edinburgh Handedness Inventory. Neuropsychologia 9, 97113.

Öst, L. G., 1989. One session treatment for specific phobias. Behav. Res. Ther. 27, 17.

Pizzagalli, D., Regard, M., Lehmann, D., 1999. Rapid emotional face processing in the human right and left brain hemispheres: An ERP study. NeuroReport 10, 26912698.

Pizzagalli, D., Lehmann, D., Koening, T., Regard, M., Pascual Marqui, R.D., 2000. Face elicited ERPs and affective attitude: Brain electric microstate and topography analyses. Clin. Neurophysiol. 111, 521531.

Rapee, R.M., Heimberg, R.G., 1997. A cognitive behavioral model of anxiety in social phobia. Behav. Res. Ther. 35, 741756. 
Schupp, H.T., Öhman, A., Junghöfer, M., Weike, A.I., Stockburger, J., Hamm, A.O., 2004. The facilitated processing of threatening faces: an ERP analysis. Emotion 4, 189200.

Schweinberger, S.R., Pickering, E.C., Jentzsch, I., Burton, A.M., Kaufmann, J.M., 2002. Event related brain potential evidence for a response of inferior temporal cortex to familiar face repetitions. Brain Res. Cogn. Brain Res. 14, 398409.

Schweinberger, S.R., Baird, L.M., Blümler, M., Kaufmann, J.M., Mohr, B., 2003. Interhemispheric cooperation for face recognition but not for affective facial expressions Neuropsychologia 41, 407414.

Schweinberger, S.R., Huddy, V., Burton, A.M., 2004. N250r A face selective brain response to stimulus repetitions. NeuroReport 15, 15011505.

Schyns, P.G., Jentzsch, I., Johnson, M., Schweinberger, S.R., Gosselin, F., 2003. A principled method for determining the functionality of brain responses. NeuroReport 14, 16651669.

Spector, I.P., Pecknold, J.C., Libman, E., 2003. Selective attentional bias related to the noticeability aspect of anxiety symptoms in generalized social phobia. J. Anxiety Disord. 17, 517531.

Stalans, L., Wedding, D., 1985. Superiority of the left hemisphere in the recognition of emotional faces. Int. J. Neurosci. 25, 219223.

Stanners, R.F., Byrd, D.M., Gabriel, R., 1985. The time it takes to identify facial expressions: effect of age, gender of subject, sex of sender, and type of expressions. J. Nonverbal Behav. 9, 201213.

Stein, M.B., Goldin, P.R., Sareen, J., Zorrilla, L.T., Brown, G.G., 2002. Increased amygdala activation to angry and contemptuous faces in generalized social phobia. Arch. Gen. Psychiatry 59, 10271034.
Straube, T., Kolassa, I. T., Glauer, M., Mentzel, H. J., Miltner, W.H.R., 2004. Effect of task conditions on brain responses to threatening faces in social phobics: an event related functional magnetic resonance imaging study. Biol. Psychiatry 56, 921930.

Stroop, J.R., 1935. Studies of interference in serial verbal reactions. J. Exp. Psychol. 18, 643662.

Tipples, J., Atkinson, A.P., Young, A.W., 2002. The eyebrow frown: a salient social signal. Emotion 2, 288296.

Turner, S.M., Beidel, D.C., Dancu, C.V., Stanley, M.A., 1989. An empirically derived inventory to measure social fears and anxiety: The Social Phobia and Anxiety Inventory. Psychol. Assess. 1, 3540.

Wells, A., Matthews, G., 1994. Attention and Emotion: A Clinical Perspective. Erlbaum, Hillsdale, NJ.

Williams, J.M.G., Mathews, A., MacLeod, C., 1996. The emotional Stroop task and psychopathology. Psychol. Bull. 120, 324.

Williams, J.M.G., Watts, F.N., MacLeod, C., Mathews, A., 1997. Cognitive Psychology and Emotional Disorders 2nd ed. John Wiley and Sons, Chichester.

Williams, L.M., Palmer, D., Liddell, B.J., Song, L., Gordon, E., 2006. The 'when' and 'where' of perceiving signals of threat versus non threat. NeuroImage 31, 458467.

Wilson, N.F., Johnston, F.E., Rosenbaum, F.F., Erlanger, H., Kossmann, C.E., Hecht, H., Cotrim, N., Menezes de Olivieira, R., Scarsi, R., Barker, P.S., 1944. The precordial electrocardiogram. Am. Heart J. 27, 1985.

Wittchen, H. U., Wunderlich, U., Gruschwitz, S., Zaudig, M., 1997. Strukturiertes Klinisches Interview für DSM IV [Structured clinical interview for DSM IV]. Hogrefe, Göttingen.

Wolpe, J., Lang, P.J., 1964. A fear survey schedule for use in behaviour therapy. Behav. Res. Ther. 2, 2730. 